\title{
Decoding osteoarthritis
}

Osteoarthritis (OA) is a

heterogeneous disease of a highly polygenic nature; genome-wide association studies (GWASs) have so far uncovered 33 genetic variants that influence the risk for the disease. A meta-analysis of genotype data from the Icelandic population from deCODE genetics and the UK Biobank now adds a further 16 loci 12 for hip and four for knee OA.

The study, published in Nature Genetics, combines data from $>17,000$ patients with hip OA and almost 24,000 with knee OA along with 562,000 individuals without $\mathrm{OA}$ as controls to analyse 11.6 million genetic variants in the largest GWAS for the disease.

Despite the previously reported (and here replicated) genetic correlation between hip and knee $\mathrm{OA}$, none of the newly identified loci overlap between the two subtypes, lending support for the idea that hip and knee OA have different genetic aetiologies.
The authors highlight a rare missense variant in SMO (involved in osteogenesis and chondrocyte differentiation) that has an odds ratio of 2.84 for increasing risk of hip OA, as well as a low-frequency missense variant in IL11 (which encodes a stimulator of osteoclast differentiation) and two independent low-frequency variants in COL11A1 (which encodes a type XI collagen) as plausible candidates for modifying risk of hip OA. A majority of the identified common variants (variants with a minor allele frequency $>5 \%$ ) are in promoter and enhancer regions of relevant cell types (such as osteoblasts and chondrocytes), a finding that is consistent with other complex traits.

The authors also use Mendelian randomization to infer direct causal effects of anthropometric traits on the risk of OA and confirm a previously reported epidemiological correlation between BMI and both hip and knee OA
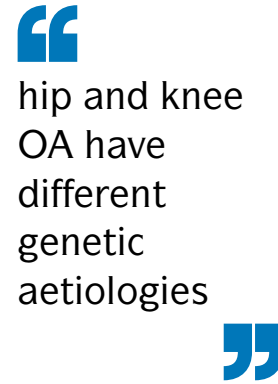

\section{OSTEOARTHRITIS}

\section{Crosstalking with Hippo}

A new study in Nature Communications identifies the transcriptional co-activator YAP as a mediator of crosstalk between Hippo and NF- $\kappa B$ signalling that is central to cartilage homeostasis and the degeneration that occurs in osteoarthritis (OA).

"This study is an extension of our previous work on the role of YAP in chondrocyte differentiation during skeletal development and bone repair," says corresponding author Kingston King-Lun Mak.

In the Hippo signalling pathway, LATS1 and LATS2 phosphorylate YAP and thereby sequester it in the cytoplasm, preventing it from translocation into the nucleus where it can affect gene expression.

In the new study, the researchers show that the NF- $\kappa B$ signalling pathway protein TAK1 can regulate YAP in the same way and tag it for proteasomal degradation.

The importance of these signalling interactions in cartilage degeneration and $O A$ is supported by in vivo data.
The researchers show that expression of YAP by chondrocytes declines with age in mice, and there is a similar reduction in patients with OA. To show that $\mathrm{YAP}$ is more than just a marker of age or cartilage degeneration, the researchers also used two surgically induced models of OA (anterior cruciate ligament transection and destabilization of the medial meniscus). After surgery, mice with Hippo signalling mediator MST1/2-deficient chondrocytes maintained higher expression of chondrocyte YAP and had less cartilage degeneration than mice with normal Hippo signalling. The same cartilage-protective effect of YAP was clear in transgenic mice in which YAP was overexpressed specifically in chondrocytes, and the reverse was true in mice in which YAP was deleted specifically from chondrocytes.

As proof of principle, the researchers also implanted inhibitor-soaked alginate beads into the joints of mice immediately after joint destabilization. Implantation of the indirect YAP activator lysophosphatidic acid and the direct YAP inhibitor verteporfin

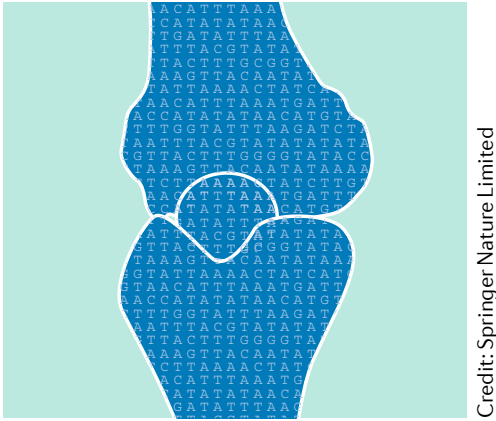

Although a number of the known 697 genetic variants for human height were also associated with $\mathrm{OA}$, the effect direction was not consistent and no genetic correlation between height and OA could be concluded, a finding that is in contrast to epidemiological studies.

With a better understanding of the genetic basis of OA (and other diseases), polygenic risk scores could be used to predict risk and subtypes of the disease in the future.

Michelle Trenkmann, Associate Editor Nature Communications

ORIGINAL ARTICLE Styrkarsdottir, U. et al. Meta-analysis of Icelandic and UK data sets identifies missense variants in SMO, IL11, COL11A1 and 13 more new loci associated with osteoarthritis. Nat. Genet. https://doi.org/10.1038/ s41588-018-0247-0 (2018)

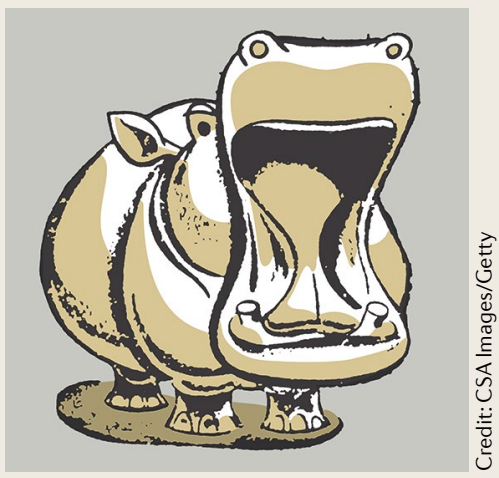

had the same effects on cartilage as overexpression and knockout of YAP, respectively.

Although this strategy of direct implantation of inhibitors is not validated in humans and these inhibitors do not naturally target the joints, it does hint that targeting Hippo signalling or otherwise promoting expression of YAP might be a viable therapeutic strategy to reduce cartilage destruction in patients with OA.

NicholasJ. Bernard

ORIGINAL ARTICLE Deng, Y. et al. Reciprocal inhibition of YAP/TAZ and NF-kB regulates osteoarthritic cartilage degradation. Nat. Commun. 9, 4564 (2018) 